\title{
Evaluación de las metodologías AICLE y EPI en el aprendizaje de los conectores en la L2
}

\author{
(Proposal for evaluation of CLIL and IPA methodologies in learning \\ connectors in a second language)
}

\author{
JuAn A. MARTínez LóPEz iD, Universidad Pablo de Olavide, Sevilla, España \\ Víctor Cantero García (iD, Universidad Pablo de Olavide, Sevilla, España
}

\author{
Volumen 6, Número 1 \\ Enero - junio de 2021 \\ p. 1 - 25
}

Este número se publicó el 4 de enero de 2021

Reseña recibida: 4 de agosto de 2020

Reseña aceptada para publicación: 11 de diciembre de 2020

ISSN: 2448-5942, https://doi.org/10.36799/el.v6i1.108

Derechos de autor: Las personas autoras conservan en todo momento sus derechos morales y patrimoniales sobre la obra; la obra no se puede alterar, transformar o ampliar; siempre debe reconocerse la autoría del documento referido. Ninguna de las modalidades de los documentos publicados en Estudios $\lambda$ ambda. Teoría y práctica de la didáctica en lengua y literatura tienen fines comerciales de naturaleza alguna.

Los contenidos de este artículo están bajo una licencia de Creative Commons Atribución No Comercial- Sin Derivadas 4.0 Internacional 


\title{
Evaluación de las metodologías AICLE y EPI en el aprendizaje de los conectores en la L2
}

\author{
(Proposal for evaluation of CLIL and IPA methodologies in learning connectors in a second \\ language)
}

\author{
JuAN A. MARTíNEZ LÓPEZ ${ }^{1}$ \\ VÍCTOR CANTERO GARCíA ${ }^{2}$
}

\begin{abstract}
RESUMEN
Uno de los apartados que mejor evidencia el grado de dominio de una lengua extranjera por parte del alumnado es su dominio de los conectores discursivos. El presente artículo tiene el objetivo de ofrecer una propuesta de evaluación para comparar los niveles de eficacia de los modelos AICLE (Aprendizaje Integrado de Contenidos y Lenguas Extranjeras) y EPI (Enfoque Plurilingüe Integrador) en cuanto a la enseñanza-aprendizaje de dichos conectores. Así, se analizan y se valoran los resultados obtenidos en la praxis del aula de dos grupos de estudiantes de nivel secundaria tras poner en la práctica dos secuencias didácticas usando sendas metodologías. Los participantes fueron 44 alumnos nativos de lenguas indoeuropeas, estudiantes del español como segunda lengua en dos escuelas de verano en Cádiz, España, en 2019. Los criterios de evaluación del uso de los marcadores son: el conocimiento de las tipologías, el o los significados de estos y la capacidad del alumnado para crear textos comprensibles, así como la riqueza de las tipologías usadas. Se creó una rúbrica como instrumento de evaluación con cuatro niveles de dominio en el uso de los conectores. La investigación se enmarca en el ámbito del modelo de intervención en el aula desde una perspectiva empírico-analítica. Los datos que arroja la evaluación de los procesos de aprendizaje a través de una y otra metodología parecen sugerir que los aprendices se apropian con mayor facilidad de los pormenores de la práctica lingüística de los conectores con el enfoque EPI que con el modelo AICLE.
\end{abstract}

PALABRAS CLAVE: conectores discursivos, bilingüismo, evaluación, rúbricas

\begin{abstract}
One of the elements that best demonstrates the degree of mastery of a foreign language by students is their mastery of discursive connectors. This article aims to offer an evaluation proposal to compare the effectiveness of the CLIL (Integrated Learning of Contents and Foreign Languages) and the IPA (Integrated Plurilingual Approach) approach in terms of teaching/learning of these connectors. Thus, the results obtained in the praxis of the classroom of two groups of secondary-level students will be analysed and evaluated after putting into practice two didactic sequences using both methodologies. The participants were 44 native Indo-European language students, students of Spanish as a second language in two summer schools in Cádiz, Spain, in 2019. The criteria for evaluating the use of markers are: the knowledge of the typologies, their meaning and the ability of the students to create understandable texts, as well as the richness of the typologies used. A rubric was created as an evaluation instrument with four levels of mastery in the use of the connectors. The research is framed within the scope of the intervention model in the classroom from an empirical-analytical perspective. The data provided by the evaluation of learning processes through both methods seem to suggest that the details of the linguistic practice of the connectors are more easily appropriated by the learners with the IPA approach than with the CLIL model.
\end{abstract}

KEY WORDS: discursive connectors, bilingualism, assessment, rubrics

${ }^{1}$ Se doctoró en Filología Española en 1996 por la Universidad de Granada, y es actualmente catedrático de Lengua Española en el Departamento de Filología y Traducción de la Universidad Pablo de Olavide (Sevilla - España). ORCID https://orcid.org/0000-00019113-7931 Correo electrónico: jamarlop@upo.es

${ }^{2}$ Es doctor en Filología Hispánica por la Universidad de Cádiz (España) desde 1994. Ha trabajado como Profesor asociado en la Universidad Pablo de Olavide (Sevilla, España), entre 2009 y 2014. Actualmente es profesor honorario en dicha universidad. ORCID https://orcid.org/0000-0003-0510-6712 Correo electrónico: vcangar@upo.es 


\section{INTRODUCCIÓN}

Una de las tareas que entraña mayor dificultad en el proceso de aprendizaje de una lengua extranjera (LE o L2) es la correcta utilización de las expresiones que pertenecen a la gramática del discurso, especialmente los marcadores discursivos y los conectores. Si como señalan Martí y Fernández (2013, 6), «la competencia en el uso de estos marcadores es signo de una madurez lingüística y comunicativa, pues son herramientas indispensables en el desarrollo de las habilidades necesarias para la construcción de los mensajes escritos y hablados», la enseñanza de dichos conectores, como parte de los contenidos curriculares, tiene gran importancia para los profesores de lengua extranjera. Resulta, por tanto, evidente que, al igual que sucede con la enseñanza del español como lengua propia, en la docencia de una L2 «es necesario sobrepasar el límite oracional para que los alumnos sean capaces de adquirir las estructuras y las estrategias discursivas que les permitan desarrollar su competencia comunicativa» (Garrido, 2000, 323). Por tanto, dada la importancia de estos elementos discursivos, su enseñanza requiere conocer las características de los aprendices y disponer de herramientas adaptadas y disponibles que faciliten la apropiación de estas unidades por parte de los hablantes (Meléndez, 2016). Así, una vez que los alumnos disponen de dichas herramientas, se hace preciso realizar una propuesta de evaluación sobre los niveles de dominio de dichos conectores, pues estas categorías proporcionan al aprendiz un modelo integral de comunicación en uso, además de ser las que nos muestran el funcionamiento de esta realidad compleja que llamamos "discurso".

El trabajo que presentamos es una experiencia en el aula cuyo objeto es medir la habilidad de dos grupos de estudiantes de español como lengua extranjera para usar los conectores en dicha lengua. Dichos grupos, con un nivel similar de español como L2, han seguido la misma unidad didáctica, si bien adaptadas a los dos paradigmas que vamos a tratar: el modelo AICLE (Aprendizaje Integrado de Contenidos y Lenguas Extranjeras; CLIL, por sus siglas en inglés) y el enfoque EPI (Enfoque Plurilingüe Integrador; IPA, por sus siglas en inglés). Así pues, nuestro objetivo es determinar cuál de los dos arquetipos mencionados arroja mayores niveles de eficiencia en cuanto a la adquisición por el aprendiz de los conectores del discurso.

Hay que tener presente que, en estos últimos años, las distintas administraciones educativas en España vienen proponiendo al profesorado diferentes modelos de educación, los cuales dependen tanto de la realidad lingüística de cada territorio, como de la interpretación que dichas administraciones hacen del concepto de biligüismo y de plurilingüismo. Entre dichos modelos destaca el ya mencionado AICLE, que es el más común en la enseñanza de segundas lenguas y que supone el aprendizaje de 
contenidos no lingǘsticos a través de una lengua distinta a la materna. Sin minusvalorar las ventajas que este modelo ha reportado a los aprendices, su protagonismo se ha reducido durante los últimos años en pro del enfoque EPI. En la actualidad ambos paradigmas coexisten, sin que hasta la fecha se cuente con estudios fiables sobre su grado de eficacia en el aprendizaje significativo de las L2 que se estudian en las aulas bilingües y plurilingües. Por tanto, pretendemos responder las siguientes cuestiones: ¿con cuál de las dos metodologías mencionadas los aprendices desarrollan de forma más exitosa su competencia comunicativa de la L2 en relación con conectores discursivos? ¿Mediante cuál de ellas los estudiantes practican con más éxito la reflexión metalingüística?

De acuerdo con lo expuesto, la presente investigación se centra en la propuesta de evaluación, mediante rúbricas, de los niveles de consecución de los objetivos fijados por el profesor en dos secuencias didácticas: una del modelo AICLE y otra del enfoque EPI, en relación con el dominio de los conectores discursivos por el aprendiz. Por medio de esta evaluación podremos comprobar con qué modelo acaban dominando de forma más exitosa el uso de dichos conectores.

\section{FUNDAMENTOS TEÓRICOS}

En este apartado procedemos a la descripción de los fundamentos teóricos que sustentan ambas metodologías, pues de ellas se derivarán las respectivas propuestas para la praxis de la evaluación de los niveles de consecución de los objetivos previstos en las secuencias didácticas propuestas para el aprendizaje de los conectores discursivos.

\subsection{La metodología AICLE}

Para encontrar una primera definición del término, nos tenemos que remontar a los primeros documentos oficiales de la UE en el que figura que «CLIL is a generic umbrella term which would encompass any activity in which a foreign language is used as a tool in the learning of a non-language subject in which both language and the subject have a joint curricular role». (Marsh, 2002, p. 58). Esta analogía con el paraguas nos indica que AICLE es algo más que un modelo. Se trata más bien de un entorno de aprendizaje en el que se implementan diferentes enfoques, de aquí que este término pueda utilizarse para nombrar:

[...] a dozen or more educational approaches (e.g. immersion, bilingual education, multilingual education, language showers and enriched language programms) [...] The flexibility of the approach is, above all, evident in the amount of time devoted 
to teaching and learning through the second language. CLIL allows for low-to highintensity exposure to teaching/learning through a second language. (Mehisto, Marsh y Frigols, 2008, p. 12)

Sin embargo, y pese a las dificultades de definir AICLE como un término unívoco, se pueden compendiar, tal y como señala Wolf $(2007$, p. 16), los tres elementos sustanciales de dicha metodología: 1. El aprendizaje de la lengua es tan importante como el del contenido. 2. Contenido y lengua se aprenden de manera integrada. 3. Aunque la lengua se utilice solo como medio de instrucción, se le presta atención cuando sea necesario para comprender mejor los contenidos.

Dichos elementos están en las raíces de un paradigma que, si bien en sus inicios se entendió como una nueva metodología para la enseñanza de lenguas (Graddol, 2006), en la actualidad sus objetivos suponen una verdadera innovación pedagógica que ha supuesto un cambio importante en la práctica de la enseñanza de las lenguas. Así, tal y como indica Moate (2011, p. 18):

CLIL is more than a methodology. Indeed the practical changes required by CLIL - the way educational activity is framed and enacted - support the notion that CLIL is methodological. The research findings, however, indicate that CLIL does not only require change in the doings of the classroom, but also in the beings of the classroom.

Estas son las características de un modelo cuyos fundamentos teóricos y presupuestos metodológicos quedaron descritos por Coyle (1999, p. 59):

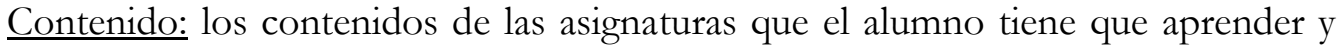
adquirir. Se basa en la progresión. Cognición: los contenidos tienen que ser pensados y entendidos, operación que se realiza mediante la lengua. Comunicación: la lengua que se usa para aprender los contenidos y la lengua que se aprende, interaccionan entre ambas realidades. Cultura: el desarrollo de la propia cultura y de las otras.

Tales fundamentos tienen una clara conexión con los componentes psicolingüísticos que intervienen en el proceso del aprendizaje de una lengua: 1) Inicio: exposición del aprendiz a un input auténtico, variado y con finalidad comunicativa, en clara alusión a la teoría de Krashen (1982 y 1985) sobre adquisición de segundas lenguas. 2) Procesar el significado: tarea que conecta con los recursos 
psicológicos de la memoria a corto y largo plazo. 3) Procesar la forma: labor a realizar de acuerdo con los modelos fijados por la psicología cognitiva. 4. Producción: la consecución del producto final que demanda del aprendiz un esfuerzo psicológico y lingüístico mayor que el exigido para comprender el imput. Sin el esfuerzo de dicha producción el itinerario del aprendizaje de una lengua queda incompleto.

\subsection{La metodología EPI}

La metodología EPI es un enfoque de la enseñanza plurilingüe donde el aprendizaje de la L2 se realiza a través de la acción. Por medio de la ejecución de tareas, el aprendiz adquiere el dominio de la lengua extranjera. Así, mientras el modelo AICLE incide en la función comunicativa de la lengua como herramienta para la realización de tareas, en el enfoque EPI el aprendiz es el agente y protagonista de los actos de habla, siendo el contexto el que le facilita los elementos necesarios para actuar, sin que el docente tenga que proporcionárselos. En definitiva, en la metodología EPI el estudiante es agente de su propio aprendizaje, puesto que, como señala Piccardo (2014, p. 12): «not only becomes a communicator engaged in the negotiation of meaning, but also takes greater responsibility for his or her learning». Tal protagonismo implica que la práctica comunicativa no sea el único recurso para dominar la L2, sino que el alumno utilice otras estrategias como la reflexión o el pensamiento crítico.

El enfoque EPI se asienta sobre la teoría sociocultural (Vigotsky, 1978), la cual sostiene que el aprendiz desarrolla su aprendizaje mediante la interacción social a través de su participación proactiva en el ambiente que le rodea, de tal modo que su desarrollo cognitivo es fruto de un proceso colaborativo. El aprendiz debe descubrir por sí mismo cómo realizar las tareas con independencia de las explicaciones del adulto. Por tanto, el papel principal del profesor es lograr que el alumno transite de un extremo al otro de la denominada "Zona de Desarrollo Próximo", es decir, que desarrolle la capacidad de comprender los conceptos e ideas que otros le muestran y explican, así como generar de forma autónoma su propio aprendizaje por medio de la interiorización, apropiación y asimilación, lo que en el caso del EPI consiste en la conceptualización translingüística. En este sentido, tal como se precisa en Esteve, Martín y Atienza (2017, p. 10), el EPI:

Was designed according to the Vygotskian principle of double stimulation, as applied by Engeström (2011). It thus aimed to encourage participants as active agents to bring about transformations in their own teaching practice. The aim of the first stimulus is to get the agents to verbalize tensions and contradictions about their own practice, as well as become aware of problems that these tensions and 
contradictions may give rise to. The aim of the second is to help overcome such problems through external mediation, involving a challenge for the agents and ultimately leading them to reconceptualize their initial understandings (Negueruela, 2011).

Esta reconceptualización es posible debido a que el EPI se basa en la corriente pedagógica del CBI (Concept Based Instruction): 1. Presentación del concepto, 2. Materialización, 3. Verbalización en sí mismo y de sus aplicaciones prácticas, 4. Automatización o interiorización (Galperin, 1992), la cual enseña al discípulo a relacionar los conceptos que ya posee, con los nuevos conceptos lingüísticos por medio de las conexiones metalingüísticas.

La segunda columna sobre la que se asienta el EPI es su concepción de la competencia plurilingüe como una realidad distinta del multilingüismo. Mientras este se refiere a la simple coexistencia de distintas lenguas en diferentes contextos sociales y educativos específicos, el EPI trabaja la competencia personal del aprendiz:

As an individual person's experience of language in its cultural contexts expands, from the language of the home to that of society at large and then to the languages of other peoples (whether learnt at school or college, or by direct experience), he or she does not keep these languages and cultures in strictly separated mental compartments, but rather builds up a communicative competence to which all knowledge and experience of language contributes and in which languages interrelate and interact. (Consejo de Europa, 2001, p. 5)

El tercer puntal de la metodología EPI es su concepción holística del lenguaje, pues asume los principios de la lingüística sistemático-funcional (Halliday, 1978); teoría que concibe la lengua como un sistema semiótico en el que la creación del significado mediante recursos lingüísticos tiene prioridad sobre la atención a la forma. Dicho sistema semiótico se materializa en el texto como unidad lingüística básica cuya carga semántica viene determinada por el propósito comunicativo, el cual debe ser completado por el estudiante al escoger los elementos que mejor le vienen a cada acto comunicativo dentro del sistema lingüístico. 


\subsection{Propuestas metodológicas para la práctica de la evaluación en AICLE y EPI}

En consonancia con el propósito del presente trabajo, procede que presentemos las distintas propuestas para el ejercicio de la evaluación de las secuencias didácticas en cada uno de los dos paradigmas contrastados. Unas propuestas que emanan del marco teórico de cada modelo y que dan lugar a postulados evaluadores distintos. Dado que AICLE:

Is conceived as an approach to education in which language teaching and subject learning are combined whit the teaching of school subjects, content and language integrated learning (CLIL) is inspired by a twofold objective. It is mean to ensure first that pupils acquire knowledge of curricular subject matter and secondly develop their competence in a language other than the normal language of instruction. (Eurydice, 2006, p. 22)

Parece lógico, por tanto, que sus propuestas metodológicas para la evaluación se encaminen a medir y cuantificar los niveles de progresión del aprendizaje, tanto de los contenidos curriculares como en el dominio de la L2. Por tanto, dicha metodología AICLE no solo se propone desarrollar el conocimiento relacionado con la materia y la capacidad de aprendizaje, estimulando la asimilación de la materia por medio de un enfoque diferente e innovador (objetivos educativos), sino las habilidades del lenguaje que enfatizan la comunicación efectiva, motivando a los alumnos a aprender idiomas usándolos para fines prácticos reales (objetivos lingüísticos). En este sentido, pude decirse que la metodología de evaluación AICLE es 1. Continua: abarca el conjunto de actividades del proceso de enseñanza-aprendizaje. 2. Sumativa: cuantifica el grado de acierto del aprendiz en la ejecución de las tareas parciales y la final. 3.- Retroalimentativa: es entendida como "teacher's feedback". Es decir, profesor y alumno se retroalimentan por medio del conocimiento de los aciertos y errores detectados. 4.- Formativa: pues ayuda a mejorar la consecución de los objetivos de aprendizaje. Promueve la reflexión para la mejora tanto del profesor como del estudiante. 5.- Por rúbricas: usa rúbricas como instrumento de medición que cuenta con criterios establecidos y estándares de desempeño por niveles y escalas. Estas características perfilan un patrón evaluador como conjunto de actuaciones cuyo objeto es la autoregulación del aprendizaje por parte del estudiante, a la vez que mide su capacidad en la realización de tareas realistas y relevantes (Monereo, 2003).

Distintos son los presupuestos que inspiran la metodología de la evaluación en EPI. En dicha metodología, como se sabe, la evaluación se concibe como un instrumento al servicio de la coconstrucción del aprendizaje (Esteve, 2002). En este caso no es el docente quien articula el proceso 
evaluador, sino que maestro y aprendiz son coparticipes en dicho proceso. El docente deja de ser medidor de conocimientos y habilidades para convertirse en guía, facilitador y orientador del aprendizaje del discípulo. Tanto docente como discente deben tener muy claro qué evaluar, por qué evaluar y cómo evaluar, asegurándose que lo evaluado se corresponde con los objetivos propuestos y que el alumno conoce con antelación de lo que se le va a evaluar y con qué criterios y herramientas.

En base a estos presupuestos, la metodología de evaluación en EPI cuenta con los siguientes rasgos: 1. Continua y formativa: referida a tareas realistas. Lo que se pretende conocer es el grado de progreso del aprendiz en la ejecución de las tareas. 2. Directa: el aprendiz actúa en la habilidad que se pretende medir. 3. Efectiva: debe describir con claridad lo que se pretende que al alumno haga. 4- En feedback: el acto evaluador proporciona a profesor y discípulo lo que se precisa mejorar y cómo hacerlo. 5.- Por rúbricas: para cotejar el grado de acierto en la realización de tareas concretas. En suma, se trata de un proceso evaluador que se aleja de lo cuantitativo para convertirse en una fuente de aprendizaje y elemento motivador para el aprendiz, gracias al cual incrementa su conocimiento metalingüístico y «adquiere una mayor conciencia de las semejanzas y diferencias entre las lenguas, sus sistemas y convenciones de uso, y, por ende, una mayor activación de sus estrategias de aprendizaje» (Martín, 2014, p. 54).

\section{METODOLOGÍA}

\subsection{Objetivos}

El presente estudio trata, como se ha indicado, de evaluar los resultados obtenidos por dos grupos de alumnos que han seguido una misma unidad didáctica, en el ámbito del español como lengua extranjera, partiendo de enfoques metodológicos, AICLE y EPI, bien diferenciados. Para llevar a cabo dicha comparación se ha recurrido a una unidad didáctica preexistente sobre la que se han realizado los cambios de diseño exigidos por cada enfoque.

El nivel de éxito en el uso de los diferentes conectores en español ha sido el eje del experimento, si bien todo se ha enmarcado en una unidad didáctica más amplia con la que se ha pretendido mejorar el conjunto de las destrezas. Dicho método se enmarca en el ámbito del modelo de intervención en el aula desde una perspectiva empírico-analítica. 


\subsection{Participantes}

Al objeto de medir la eficacia de una y otra metodología en el contexto de la didáctica de los marcadores pragmáticos, se nos presentó la posibilidad de poner práctica la misma unidad didáctica en dos grupos diferentes de estudiantes europeos de español como lengua extranjera.

El primero de los grupos referidos lo componían 22 alumnos estudiantes de español como L2 oriundos de Holanda, que durante el verano de 2019 tuvieron una estancia de un mes en una escuela de verano en El Puerto de Santa María (Cádiz-España). La edad del grupo, formado por diez chicas y doce chicos, era de 15 y 16 años. El nivel de dichos estudiantes, tras cuatro años de estudio de español como lengua extranjera era B1-B2. El segundo grupo estaba formado por 20 alumnos noruegos que, durante ese mismo verano, tuvieron una estancia de estudios en Jerez de la Frontera (Cádiz-España). Dicho grupo estaba compuesto por 11 chicas y 9 chicos de edades muy similares a las del grupo anterior. El nivel de español de dicho grupo, después de 7 semestres de estudio en su país, estaba dentro de los márgenes del B1-B2. Así pues, ambos grupos procedían del entorno cultural europeo, nativos de lenguas indoeuropeas y con niveles de español bastante similares.

Dichos grupos siguieron, como ya se ha señalado, la misma unidad didáctica; si bien, mientras el primer grupo, el de holandeses, siguió la unidad didáctica adaptada al modelo AICLE, el segundo grupo, el de los noruegos, siguieron la misma unidad adaptada al modelo EPI.

\subsection{Presentación de la unidad didáctica}

La secuencia didáctica elegida para dicho experimento, que deseábamos relacionase las materias de Literatura española y Gramática, fue Silencio: La casa de Bernarda Alba, cuyo autor es Jorge Berenguer (2013). Su puesta en práctica se desarrolló durante tres días, dedicando dos horas diarias a dicha secuencia. Siguiendo los objetivos planteados desde el inicio, mucho del material de dicha secuencia estaba orientado a la didáctica de los marcadores pragmáticos y concretamente a los conectores.

En el cuadro 1 se recogen los objetivos de aprendizaje y los contenidos, así como algunos de los ejercicios propuestos.

\section{Cuadro 1.}

Objetivos de aprendizaje y contenido de la unidad didáctica:

"Silencio de la Casa de Bernarda Alba"

\begin{tabular}{|l|l|}
\hline I. Objetivos de aprendizaje & II. Contenidos de material \\
$\begin{array}{l}\text { 1. Acercarse a la producción dramática de Federico } \\
\text { García Lorca. }\end{array}$ & $\begin{array}{l}\text { 1. Literarios: La casa de Bernarda Alba } \\
\text { (fragmentos): Acto I "Bernarda declara }\end{array}$ \\
\hline
\end{tabular}


2. Desarrollar la competencia en comunicación lingüística en la comprensión y análisis de los textos propuestos y en la composición y revisión de los propios utilizando un registro adecuado. Potenciar en el alumno el uso adecuado de los conectores argumentativos, conectores

3. de relaciones lógica y organizadores del

4. discurso.

5. Profundizar en los valores simbólicos presentes en la escritura literaria.

6. Caracterizar el habla de un personaje. ocho años de luto en la casa"; acto III "Discusión de Adela y Martirio y final trágico" (Federico García Lorca).

2. Gramaticales:

- Uso de los artículos determinados e indeterminados.

- Conectores argumentativos:

a) Refuerzo: además, encima, incluso, igualmente, también, asimismo, del mismo modo, en realidad, de hecho.

b) Ejemplificación: por ejemplo, a saber, etc.

c) Contraste: pero, en cambio, sin embargo, ahora bien, por el contrario.

- Conectores de relaciones lógicas:

a) De causa: porque, por eso, puesto que...

b) De consecuencia: por tanto, de abi que...

c) De condición: si, a no ser que, a menos que...

d) De finalidad: para qué, a fin de qué...

- Organizadores del discurso:

a) Ordenación secuencial: para empezar, en primer lugar, por un lado, por una parte.

b) Reformulación: o sea, es decir, esto es, en otras palabras, mejor dicho, más bien.

c) Introducción de un tema: por lo que respecta a, en cuanto a, por lo que se refiere a.

d) Digresión (cambio de tema): por cierto, a propósito, a todo esto, hablando de otra cosa.

3 Léxicos: ampliación del vocabulario ya conocido por el alumno.

- La expresión de la obligación, opinar y argumentar.

- Expresar acuerdo, acuerdo parcial o desacuerdo.

\section{Contenido Lengua/ Comunicación. Vocabulario.}

1. Lenguaje general relacionado con la obra literaria estudiada que sirva de ampliación de su conocimiento léxico de B2.

2. Lenguaje técnico de la materia: metáfora, hipérbole, simbolismo, comparación, acto y otras palabras del campo léxico.

\section{Estructuras}

1. Estructuración de la argumentación mediante conectores discursivos: aditivos (además, tampoco, incluso, asimismo, también, de la misma manera, etc.), contraargumentativos (en cambio, por el contrario, antes bien, sin embargo, no obstante, ahora bien, etc.), aclaratorios (es decir, en otras palabras, mejor dicho), consecutivos (por lo tanto, en consecuencia, por eso, etc.) y conclusivos

(Finalmente, en resumen, en sintesis, en definitiva, etc.)

2. Conectores de relaciones lógicas

3. Conectores de ordenación secuencial

4. Ausencia y uso del artículo determinado e indeterminado.

5. Expresar acuerdo, acuerdo parcial o desacuerdo: 


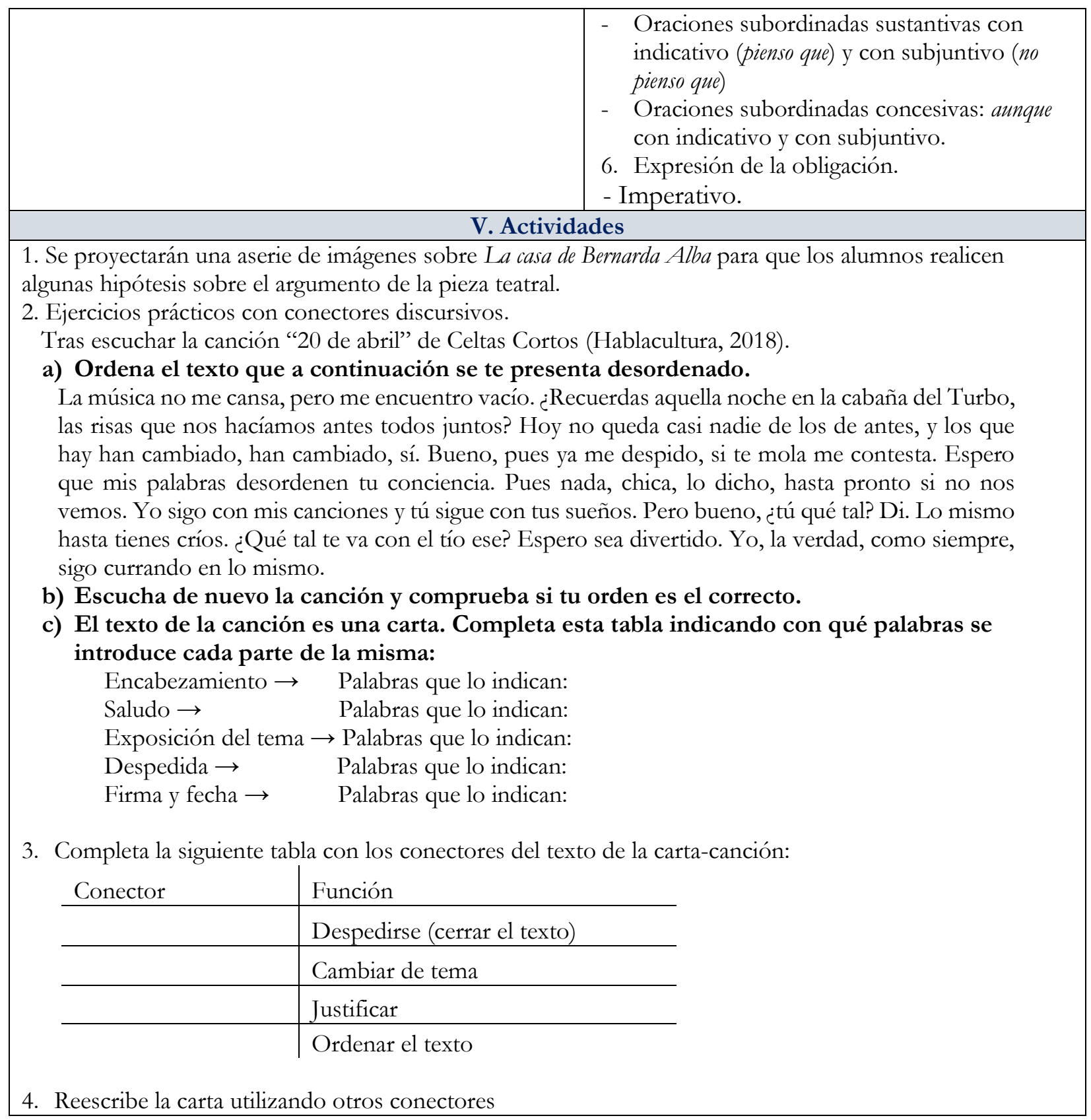

Fuente: Elaboración propia, a partir de la propuesta de Jorge Berenguer (2013)

\subsection{Adaptación de la unidad didáctica}

El trabajo con el primer grupo, con el que se pretendió la adaptación metodológica al concepto AICLE, se desarrolló en un contexto meramente bilingüe utilizando en gran medida el español, pero con un cierto apoyo de su lengua materna, el holandés. La unidad didáctica se presenta compartimentada en dos áreas: literatura española a partir de la obra literaria de Federico García 
Lorca y otra de Gramática, en la que se pretende que los alumnos vean la importancia del uso de los conectores en el proceso comunicativo. En este sentido hemos de recalcar que el sistema AICLE tiene como objeto el alcanzar una competencia lingüística en una lengua extranjera a la vez que se adquiere competencia en áreas vinculadas a otras áreas como la economía, la literatura o las ciencias naturales (Bludau, 1996).

Para la realización de actividades se trabajó de modo individual, si bien en algunos casos se formaron grupos pequeños con la finalidad de que ellos, en grupo, diesen solución a los problemas planteados. Así, salvo algunas leves modificaciones realizadas por cuestiones meramente prácticas, se respetó gran parte de la unidad tal y como estaba diseñada. También se tuvieron en cuenta las habilidades lingüísticas básicas de AICLE, particularmente las comprensiones auditiva y lectora, y la expresión verbal. Un aspecto de este enfoque, que también se tuvo en cuenta es el mantener al mismo nivel tanto los contenidos lingüísticos como los no lingüísticos (Coyle, 2006).

El grupo de estudiantes noruegos de español como lengua extranjera siguió, como se ha señalado, la misma unidad didáctica, pero modificada en ciertos aspectos para acercarla a la metodología del Enfoque Plurilingüe Integrador. En este sentido, de cara al aprendizaje del uso de los marcadores se fomentó entre los estudiantes la posibilidad de trabajar y comparar la tipología y comportamiento de dichos elementos en otras lenguas que la práctica totalidad del grupo conocía, como eran el inglés y el alemán. En relación con esto, algunos autores han señalado que «cada persona plurilingüe cuenta con diversas compilaciones mentales previstas que le posibilitan actuar en diferentes entornos lingüístico-culturales con distintos grados de dominio» (Mallol y Alsina, 2017, p. 46).

Por otra parte, siguiendo las pautas del modelo EPI, se ha pretendido que el aprendizaje debe integrar todas las áreas posibles, en nuestro caso: la lengua, la literatura, la historia, la cultura..., si bien se ha puesto énfasis en el uso adecuado de los conectores para articular un discurso eficaz. Se trata, en esencia, de «tratar la lengua como una realidad discursiva inscrita en contextos sociales y culturales» (Martín, 2014, p. 56). Para ello, se fomentó una participación más activa de los estudiantes de modo que pudiesen contrastar los aspectos tratados (culturales, literarios y lingüísticos) con sus experiencias y lo aprendido sobre otras lenguas y culturas, incluida su propia lengua materna.

Otro de los aspectos más relevantes de esta experiencia fue el fomentar que el aprendiz fuese en mayor grado agente de su propio aprendizaje. Para ello se dio a los alumnos, así como a 
los grupos formados por ellos, una mayor autonomía y capacidad para decidir las prácticas, tanto en su orden como en el desarrollo de estas.

Los docentes, tanto los españoles como los noruegos, tuvieron que coordinarse al objeto de que los contenidos se complementasen para aprovechar todos los conocimientos previos de los aprendices y, por tanto, favorecer un aprendizaje rápido, activo y significativo.

Este enfoque también nos ha permitido diseñar tareas con el fin de favorecer el usoaprendizaje reflexivo de la lengua (Van Lier, 2007). Tal uso reflexivo se ha pretendido fortalecer mediante actividades de tipo language awareness. Para dichas actividades se ha tomado en consideración dos dimensiones relativas al tratamiento pedagógico de la conciencia lingǘstica. Dichas dimensiones, como señala Martín (2007), son, por un lado, la temporal, «relativa al momento de la intervención didáctica en la que se trabaja la conciencia lingüística [...] mediante distintos procesos o ejercicios: antes del uso comunicativo, en el uso y tras el uso» (p.56); por otro, la derivada del carácter plurilingüe del proyecto. Sin embargo, no se trata de «una conciencia sobre el lenguaje humano en abstracto, sino una conciencia sobre fenómenos concretos de las distintas lenguas que componen el repertorio lingüístico del aprendiz» (p.56).

\subsection{Evaluación}

Para la evaluación de los procesos de aprendizaje de cada grupo, se propuso inicialmente una serie de actividades y estrategias. A partir de los contenidos recogidos en la unidad didáctica se desarrollaron una serie de criterios y un conjunto de objetivos de cara a la evaluación. Pese a que el contexto de la unidad didáctica es inicialmente de carácter literario, también en ella se trabajan las diferentes tipologías y significados de los conectores discursivos. La evaluación se basará casi exclusivamente en el correcto uso de dichos marcadores. Los criterios serán, en este sentido: el conocimiento de las tipologías, el o los significados de estos y la capacidad del alumno para crear textos comprensibles, así como la riqueza de las tipologías usadas.

De modo sucinto, al final de esta unidad el alumno debía ser capaz de:

1. Conocer e identificar los rasgos más importantes de La casa de Bernarda Alba.

2. Enunciar y analizar las características más relevantes de un personaje literario. 
3. Reflexionar sobre un tema de actualidad argumentado su punto y de vista, contrastarlo con el de sus compañeros y contraargumentar.

4. Producir textos, tanto orales como escritos, utilizando un registro adecuado y con corrección gramatical, léxica y pragmática.

Así, para el grupo adaptado al entorno AICLE se planteó a los estudiantes una evaluación formativa durante tres días que duró el experimento. La mayoría de los ejercicios realizados tras las explicaciones teóricas y el debate, y especialmente aquellos que se realizaron en el en el entorno de los marcadores discursivos, fueron de carácter individual. En ellos se intentó trabajar con las cuatro destrezas, si bien predominaron las relativas a la comunicación oral. Así mismo, también se establecieron varios grupos reducidos (tres o cuatro alumnos) cuyo fin fue realizar una tarea y fomentar el aprendizaje colaborativo en el ámbito de la resolución de problemas, que también tuvo carácter evaluativo. Al final, los estudiantes realizaron una prueba de evaluación formal que consistió en una parte escrita con dos ejercicios: el primero, escrito, que consistió en una redacción libre en la que se pedía utilizar tantos conectores como fuese posible; y un segundo ejercicio para el relleno de huecos, donde el alumno debía añadir el conector o conectores correctos en ese contexto comunicativo. El segundo ejercicio fue una breve conversación entre profesor y alumno en la que este último debía llevar a la práctica oral aquello que habían estado trabajando durante las seis horas que duró la unidad didáctica en cuestión. Seguimos, por tanto, los postulados del enfoque AICLE, para quien la evaluación no es algo que se realice tras la instrucción, sino forma parte indispensable de esta (Llinares, Morton y Whittaker, 2012).

Con respecto al grupo cuya docencia fue adaptada al entorno EPI, la evaluación se pretendió que fuese continua y formativa. Se consideró, por tanto, la evaluación como una actividad más del aprendizaje. El contexto de la unidad didáctica es de carácter literario, aunque también en una parte más lingüística se trabajó con las diferentes tipologías y significados de los conectores discursivos. La evaluación, en lo que afecta a este trabajo, se basó casi exclusivamente en el correcto uso de dichos marcadores. En este entorno pedagógico la intervención del profesor fue menor y aumento la interacción profesor-alumno. El aprendizaje se llevó a cabo mediante una tarea en grupos de cuatro alumnos, en la que el profesor tenía el papel de mero colaborador para hacer más fluida y dinámica la elaboración de dicho trabajo cooperativo. Para su realización se tuvo en consideración el uso y contraste del papel de los conectores en las otras lenguas, sus semejanzas y diferencias, pues el papel de las otras lenguas como elementos didácticos es fundamental en este enfoque. La interacción en 
tándem de ejercicios de diverso tipo, en ocasiones elegidos por ellos mismos, también fue evaluado por los dos participantes. Por otra parte, dado el importante papel de la agentividad en este enfoque, los alumnos realizaron su propia evaluación en alguna de las pruebas de carácter individual. Finalmente, al acabar el periodo dedicado a la unidad didáctica se realizó un ejercicio similar al realizado en el grupo AICLE. En el grupo EPI, como se ha indicado, la participación del alumnado en el proceso didáctico fue más activa, tanto a la hora de dirigir en cierta medida su aprendizaje como en el proceso de evaluación

\subsection{Diseño de rúbricas para la evaluación}

Las rúbricas de evaluación, como indican Gatica y Uribarren (2013), son tablas donde se describen tanto los estándares de aprendizaje evaluables, como los indicadores de logro y calidad de forma graduada para evaluar una tarea, objetivo o competencia. Así, en líneas generales, puede decirse que las rubricas facilitan la evaluación del alumnado, la elaboración de un proyecto o tarea en varios niveles de rendimiento (al objeto de aclarar lo que se espera del trabajo del alumno), y ofrecen una retroalimentación. En algunos casos, va incluso más allá, pues como señala Andrade (2000, p. 13), la rúbrica puede «help teachers justify to parents and others the grades that they assign to students. At their very best, rubrics are also teaching tools that support student learning and the development of sophisticated thinking skills».

Además de lo señalado, las rúbricas fomentan el aprendizaje constructivo (al obtener mayor retroalimentación) y la autoevaluación. Por otra parte, se basan en estándares de aprendizaje evaluables fruto de la concreción de criterios objetivos, y, lógicamente, en relación con unos determinadas competencias y contenidos. Por último, puede decirse que la rúbrica es un instrumento de clara utilidad, pues ofrece información sobre la eficacia en el proceso de aprendizaje, de la metodología en uso y de los recursos utilizados.

Para la realización de las rúbricas, como ya señalaron Wylie y Lyon:

[...] learning goals should be clearly identified and communicated to students, and should help students make connections among lessons within a larger sequence. Learning goals should be aligned to state or district grade-level standards, although this dimension focuses on how the teacher identifies the learning goals for a particular 
lesson, communicates them to the students, and uses them in a way that supports learning (2013, p. 29).

Así pues, convencidos de la utilidad de la rúbrica tanto como elemento didáctico como de evaluación, nos hemos decantado por este sistema para evaluar los aprendizajes. Para la elaboración de la rúbrica y en lo referente a los conectores hemos tomado como base la clasificación que aparece en la página de Lingolia (2020). La rúbrica utilizada en los dos grupos de alumnos es la que se puede apreciar en la tabla 2:

Tabla 2.

Propuesta de rúbrica para evaluación de uso de conectores

\begin{tabular}{|c|c|c|c|c|}
\hline Conceptos evaluados & $\begin{array}{l}\text { Nivel alto de } \\
\text { dominio }\end{array}$ & $\begin{array}{l}\text { Nivel medio de } \\
\text { dominio }\end{array}$ & $\begin{array}{l}\text { Nivel suficiente } \\
\text { de dominio }\end{array}$ & $\begin{array}{l}\text { Nivel bajo de } \\
\text { dominio }\end{array}$ \\
\hline $\begin{array}{l}\text { Conectores aditivos: } \\
\text { además, también, encima, es } \\
\text { más, etc. }\end{array}$ & $\begin{array}{l}\text { Identifica y usa bien } \\
\text { los conectores en los } \\
\text { diálogos, debates y } \\
\text { en la redacción de } \\
\text { textos }\end{array}$ & $\begin{array}{l}\text { Reconoce todos } \\
\text { los conectores, } \\
\text { pero solo } \\
\text { utiliza unos pocos } \\
\text { y siempre los } \\
\text { mismos }\end{array}$ & $\begin{array}{l}\text { Conoce tan solo } \\
\text { Algunos } \\
\text { conectores y no } \\
\text { siempre los usa } \\
\text { correctamente }\end{array}$ & $\begin{array}{l}\text { Comienza a } \\
\text { conocer y } \\
\text { utilizar algunos } \\
\text { conectores, pero le } \\
\text { cuesta usarlos bien }\end{array}$ \\
\hline $\begin{array}{l}\text { Conectores } \\
\text { contraargumentativos: en } \\
\text { cambio, al contrario, ahora } \\
\text { bien, etc. }\end{array}$ & $\begin{array}{l}\text { Realiza un uso fluido } \\
\text { y variado de estos } \\
\text { conectores. Razona } \\
\text { su uso en cada caso }\end{array}$ & $\begin{array}{l}\text { Ejecuta un buen } \\
\text { uso de estos } \\
\text { conectores, pero } \\
\text { no siempre acierta } \\
\text { a justificarlo }\end{array}$ & $\begin{array}{l}\text { Usa estos } \\
\text { conectores, pero } \\
\text { confunde sus } \\
\text { matices }\end{array}$ & $\begin{array}{l}\text { Tiene problemas } \\
\text { para diferenciar } \\
\text { diferentes tipos }\end{array}$ \\
\hline $\begin{array}{l}\text { Conectores } \\
\text { consecutivos: por tanto, asi } \\
\text { pues, por consiguiente, en } \\
\text { consecuencia, etc. }\end{array}$ & $\begin{array}{l}\text { Utiliza correctamente } \\
\text { estos conectores en } \\
\text { sus intervenciones y } \\
\text { sabe justificar su uso }\end{array}$ & $\begin{array}{l}\text { Hace un uso } \\
\text { acertado de estos } \\
\text { conectores, pero } \\
\text { los repite en varias } \\
\text { ocasiones }\end{array}$ & $\begin{array}{l}\text { Le cuesta distinguir } \\
\text { entre conectores } \\
\text { consecutivos y } \\
\text { concesivos }\end{array}$ & $\begin{array}{l}\text { Empieza a } \\
\text { distinguir } \\
\text { entre estos dos } \\
\text { tipos de } \\
\text { conectores. No } \\
\text { sabe justificar su } \\
\text { uso }\end{array}$ \\
\hline $\begin{array}{l}\text { Conectores explicativos: } \\
\text { o sea, es decir, esto es, en otras } \\
\text { palabras, etc. }\end{array}$ & $\begin{array}{l}\text { Selecciona con } \\
\text { acierto este tipo de } \\
\text { conectores } \\
\text { en cada ítem de la } \\
\text { conversación }\end{array}$ & $\begin{array}{l}\text { Utiliza con acierto } \\
\text { estos } \\
\text { conectores, pero } \\
\text { no en todos los } \\
\text { casos }\end{array}$ & $\begin{array}{l}\text { Se equivoca al usar } \\
\text { este tipo de } \\
\text { conectores, le } \\
\text { cuesta adecuar el } \\
\text { que se precisa en } \\
\text { cada ítem }\end{array}$ & $\begin{array}{l}\text { Comienza a } \\
\text { distinguir } \\
\text { este tipo de } \\
\text { conectores } \\
\text { del resto, pero falla } \\
\text { en su uso correcto }\end{array}$ \\
\hline $\begin{array}{l}\text { Conectores concesivos: } \\
\text { aun así, con todo, de cualquier } \\
\text { manera, en todo caso, etc. }\end{array}$ & $\begin{array}{l}\text { Acierta en el uso de } \\
\text { estos conectores. }\end{array}$ & $\begin{array}{l}\text { Tiene éxito en el } \\
\text { uso de estos } \\
\text { conectores, pero } \\
\text { en ocasiones no } \\
\text { sabe diferenciarlos } \\
\text { del resto }\end{array}$ & $\begin{array}{l}\text { No replica con } \\
\text { presteza a los } \\
\text { argumentos de sus } \\
\text { interlocutores }\end{array}$ & $\begin{array}{l}\text { No le resulta } \\
\text { sencillo } \\
\text { Intervenir en los } \\
\text { turnos de réplica }\end{array}$ \\
\hline $\begin{array}{l}\text { Conectores } \\
\text { reformuladores: dicho de } \\
\text { otro modo, mejor dicho, } \\
\text { bablando en plata, etc. }\end{array}$ & $\begin{array}{l}\text { Identifica y usa con } \\
\text { corrección estos } \\
\text { conectores }\end{array}$ & $\begin{array}{l}\text { Hace un uso } \\
\text { preciso de estos } \\
\text { conectores, pero } \\
\text { no siempre justifica } \\
\text { adecuadamente su } \\
\text { empleo }\end{array}$ & $\begin{array}{l}\text { Los Identifica en } \\
\text { ciertas ocasiones, } \\
\text { pero no sabe } \\
\text { usarlos de modo } \\
\text { correcto en el } \\
\text { discurso }\end{array}$ & $\begin{array}{l}\text { Empieza a } \\
\text { identificar } \\
\text { estos conectores, } \\
\text { pero no acierta a } \\
\text { usarlos en los actos } \\
\text { discursivos }\end{array}$ \\
\hline
\end{tabular}




\begin{tabular}{|c|c|c|c|c|}
\hline $\begin{array}{l}\text { Conectores } \\
\text { ejemplificativos: así, por } \\
\text { ejemplo, verbigracia, etc. }\end{array}$ & $\begin{array}{l}\text { No duda en ninguna } \\
\text { ocasión en el uso de } \\
\text { estos conectores }\end{array}$ & $\begin{array}{l}\text { Identifica este tipo } \\
\text { de conectores, } \\
\text { pero en ocasiones } \\
\text { no acierta en su } \\
\text { uso. }\end{array}$ & $\begin{array}{l}\text { Reconoce este tipo } \\
\text { de conectores tan } \\
\text { solo en algunas } \\
\text { ocasiones. casi } \\
\text { siempre falla en su } \\
\text { uso. }\end{array}$ & $\begin{array}{l}\text { Empieza a } \\
\text { distinguir cuáles } \\
\text { corresponden al } \\
\text { hablante y al } \\
\text { oyente }\end{array}$ \\
\hline $\begin{array}{l}\text { Conectores rectificativos: } \\
\text { más bien, mejor dicho, etc. }\end{array}$ & $\begin{array}{l}\text { Acierta siempre que } \\
\text { usa estos conectores, } \\
\text { sabe razonar el uso. }\end{array}$ & $\begin{array}{l}\text { Elige } \\
\text { adecuadamente } \\
\text { Estos conectores, } \\
\text { pero no acierta al } \\
\text { justificar su empleo }\end{array}$ & $\begin{array}{l}\text { En contadas } \\
\text { ocasiones hace un } \\
\text { uso correcto de } \\
\text { estos conectores. } \\
\text { No sabe justificar } \\
\text { su uso }\end{array}$ & $\begin{array}{l}\text { No tiene clara la } \\
\text { noción de } \\
\text { inferencia. } \\
\text { comienza a ubicar } \\
\text { estos conectores } \\
\text { en los ítems que } \\
\text { los precisan }\end{array}$ \\
\hline $\begin{array}{l}\text { Conectores } \\
\text { recapitulativos: al fin y al } \\
\text { cabo, en definitiva, en resumen, } \\
\text { total, etc. }\end{array}$ & $\begin{array}{l}\text { Logra utilizar con } \\
\text { corrección las } \\
\text { partículas discursivas } \\
\text { que manifiestan la } \\
\text { actitud del hablante }\end{array}$ & $\begin{array}{l}\text { Usa con fluidez } \\
\text { estas partículas, } \\
\text { pero en ocasiones } \\
\text { no distingue la } \\
\text { posición que ocupa } \\
\text { el hablante y el } \\
\text { oyente en el } \\
\text { discurso }\end{array}$ & $\begin{array}{l}\text { Tan solo en } \\
\text { contadas ocasiones } \\
\text { usa bien estas } \\
\text { partículas. } \\
\text { Confunde su uso al } \\
\text { alternar hablante y } \\
\text { oyente }\end{array}$ & $\begin{array}{l}\text { Usa repetidamente } \\
\text { Los mismos } \\
\text { conectores }\end{array}$ \\
\hline $\begin{array}{l}\text { Conectores de } \\
\text { ordenación: antes de nada, } \\
\text { en primer lugar, previamente, } \\
\text { finalmente, etc. }\end{array}$ & $\begin{array}{l}\text { Identifica y usa bien } \\
\text { los conectores en los } \\
\text { diálogos, debates y } \\
\text { en la redacción de } \\
\text { textos. }\end{array}$ & $\begin{array}{l}\text { Reconoce todos } \\
\text { los conectores, } \\
\text { pero solo utiliza } \\
\text { unos pocos y } \\
\text { siempre los } \\
\text { mismos }\end{array}$ & $\begin{array}{l}\text { Conoce tan solo } \\
\text { algunos conectores } \\
\text { y no siempre los } \\
\text { usa correctamente }\end{array}$ & $\begin{array}{l}\text { Comienza a } \\
\text { conocer y } \\
\text { utilizar algunos } \\
\text { conectores, pero le } \\
\text { cuesta usarlos bien }\end{array}$ \\
\hline $\begin{array}{l}\text { Conectores de apoyo } \\
\text { argumentativo: pues bien, } \\
\text { dicho esto, en vista de aquello, } \\
\text { etc. }\end{array}$ & $\begin{array}{l}\text { Utiliza correctamente } \\
\text { estos conectores en } \\
\text { sus intervenciones y } \\
\text { sabe justificar su uso }\end{array}$ & $\begin{array}{l}\text { Hace un uso } \\
\text { acertado } \\
\text { de estos } \\
\text { conectores, } \\
\text { pero los repite en } \\
\text { varias } \\
\text { ocasiones }\end{array}$ & $\begin{array}{l}\text { Le cuesta distinguir } \\
\text { entre conectores } \\
\text { de ejemplificación } \\
\text { y de secuenciación }\end{array}$ & $\begin{array}{l}\text { Comienza a } \\
\text { distinguir este tipo } \\
\text { de conectores del } \\
\text { resto, pero falla en } \\
\text { su uso correcto }\end{array}$ \\
\hline $\begin{array}{l}\text { Conectores de digresión: } \\
\text { por cierto, a propósito, dicho sea } \\
\text { de paso, etc. }\end{array}$ & $\begin{array}{l}\text { No duda en ninguna } \\
\text { ocasión en el uso de } \\
\text { estos conectores }\end{array}$ & $\begin{array}{l}\text { Tiene éxito en el } \\
\text { uso de estos } \\
\text { conectores, pero } \\
\text { en ocasiones no } \\
\text { sabe diferenciar. }\end{array}$ & $\begin{array}{l}\text { No replica con } \\
\text { presteza a los } \\
\text { argumentos de sus } \\
\text { interlocutores }\end{array}$ & $\begin{array}{l}\text { No le resulta } \\
\text { sencillo } \\
\text { Intervenir en los } \\
\text { turnos de réplica }\end{array}$ \\
\hline
\end{tabular}

Fuente: Elaboración propia a partir de la estructura desarrollada en Lingolia (2020)

\section{ANÁLISIS DE RESULTADOS Y DISCUSIÓN}

Una vez aplicadas las consiguientes rúbricas y después analizar los datos obtenidos tras la aplicación de pruebas de autoevaluación y comprobación de los niveles de aprendizaje de los aprendices, procedemos a la valoración de los resultados. Dicho análisis se efectúa teniendo en cuenta que, al margen de los ejercicios de evaluación, también se ha tenido en consideración otros instrumentos evaluadores utilizados por los docentes: la observación participativa del alumno a lo largo de proceso 
por medio del diario de aula y de los registros audiovisuales. Para las pruebas correspondientes a la unidad didáctica de cada grupo se han seleccionado una serie de conectores teniendo en cuenta no sólo su tipo, sino también su frecuencia de uso, de modo que se le ha asignado mayor penalización al desconocimiento o error de un conector frecuente que uno poco frecuente. Lógicamente, también se ha tenido en cuenta el nivel dónde nos hallamos y las exigencias de este, esto es, B1-B2 para ambos grupos.

Los resultados de la evaluación de la secuencia desarrollada con el modelo AICLE se pueden observar en la tabla 3:

Tabla 3.

Resultados obtenidos con el modelo AICLE.

\begin{tabular}{|c|c|c|c|c|}
\hline Conceptos evaluados & $\begin{array}{l}\text { Nivel alto } \\
\text { de dominio }\end{array}$ & $\begin{array}{l}\text { Nivel medio } \\
\text { de dominio }\end{array}$ & $\begin{array}{l}\text { Nivel suficiente } \\
\text { de dominio }\end{array}$ & $\begin{array}{l}\text { Nivel bajo } \\
\text { de dominio }\end{array}$ \\
\hline $\begin{array}{l}\text { Conectores aditivos: además, } \\
\text { también, encima, es más, etc. }\end{array}$ & $16,1 \%$ & $38,2 \%$ & $32,1 \%$ & $13,6 \%$ \\
\hline $\begin{array}{l}\text { Conectores contraargumentativos: } \\
\text { en cambio, al contrario, abora bien, etc. }\end{array}$ & $11,2 \%$ & $28,1 \%$ & $37,5 \%$ & $23,2 \%$ \\
\hline $\begin{array}{l}\text { Conectores consecutivos: por tanto, } \\
\text { asi pues, por consiguiente, etc. }\end{array}$ & $19,7 \%$ & $28,0 \%$ & $28,4 \%$ & $23,9 \%$ \\
\hline $\begin{array}{l}\text { Conectores explicativos: o sea, es } \\
\text { decir, esto es, en otras palabras, etc. }\end{array}$ & $17,5 \%$ & $29,2 \%$ & $32,1 \%$ & $21,2 \%$ \\
\hline $\begin{array}{l}\text { Conectores concesivos: aun así, con } \\
\text { todo, de cualquier manera, etc. }\end{array}$ & $13.3 \%$ & $25.0 \%$ & $32,1 \%$ & $29,6 \%$ \\
\hline $\begin{array}{l}\text { Conectores reformuladores: dicho } \\
\text { de otro modo, mejor dicho, etc. }\end{array}$ & $18,7 \%$ & $25,4 \%$ & $30,8 \%$ & $25,1 \%$ \\
\hline $\begin{array}{l}\text { Conectores ejemplificativos: así, } \\
\text { por ejemplo, verbigracia, etc. }\end{array}$ & $28,3 \%$ & $35,5 \%$ & $21,1 \%$ & $15,1 \%$ \\
\hline $\begin{array}{l}\text { Conectores rectificativos: más bien, } \\
\text { mejor dicho, etc. }\end{array}$ & $18,0 \%$ & $28,4 \%$ & $33,5 \%$ & $20,1 \%$ \\
\hline $\begin{array}{l}\text { Conectores recapitulativos: al fin y } \\
\text { al cabo, en definitiva, en resumen, etc. }\end{array}$ & $20,2 \%$ & $24,6 \%$ & $33,7 \%$ & $21,5 \%$ \\
\hline $\begin{array}{l}\text { Conectores de ordenación: antes de } \\
\text { nada, en primer lugar, finalmente, etc. }\end{array}$ & $23,2 \%$ & $30,2 \%$ & $24,1 \%$ & $22,5 \%$ \\
\hline $\begin{array}{l}\text { Conectores de apoyo } \\
\text { argumentativo: pues bien, dicho esto, en } \\
\text { vista de aquello, etc. }\end{array}$ & $17,0 \%$ & $30,4 \%$ & $36,5 \%$ & $16,1 \%$ \\
\hline $\begin{array}{l}\text { Conectores de digresión: por cierto, } \\
\text { a propósito, dicho sea de paso, etc. }\end{array}$ & $20,3 \%$ & $32,3 \%$ & $23,1 \%$ & $24,3 \%$ \\
\hline TOTAL & 223,5 & 355,3 & 365 & 256,2 \\
\hline
\end{tabular}

Fuente: Elaboración propia

Por otra parte, en la tabla 4, se muestran los resultados obtenidos por los alumnos que han seguido durante el desarrollo de la secuencia didáctica la metodología del modelo EPI. 
Tabla 4.

Resultados obtenidos con el modelo EPI.

\begin{tabular}{|c|c|c|c|c|}
\hline Conceptos evaluados & $\begin{array}{l}\text { Nivel alto } \\
\text { de dominio }\end{array}$ & $\begin{array}{l}\text { Nivel medio } \\
\text { de dominio }\end{array}$ & $\begin{array}{l}\text { Nivel suficiente } \\
\text { de dominio }\end{array}$ & $\begin{array}{l}\text { Nivel bajo } \\
\text { de dominio }\end{array}$ \\
\hline $\begin{array}{l}\text { Conectores aditivos: además, } \\
\text { también, encima, es más, etc. }\end{array}$ & $18,2 \%$ & $38,1 \%$ & $32,4 \%$ & $11,3 \%$ \\
\hline $\begin{array}{l}\text { Conectores contraargumentativos: } \\
\text { en cambio, al contrario, ahora bien, etc. }\end{array}$ & $12,1 \%$ & $32,3 \%$ & $34 \%$ & $21,6 \%$ \\
\hline $\begin{array}{l}\text { Conectores consecutivos: por tanto, } \\
\text { asi pues, por consiguiente, etc. }\end{array}$ & $19,0 \%$ & $34,0 \%$ & $24,7 \%$ & $23,2 \%$ \\
\hline $\begin{array}{l}\text { Conectores explicativos: o sea, es } \\
\text { decir, esto es, en otras palabras, etc. }\end{array}$ & $18,7 \%$ & $31,6 \%$ & $33,4 \%$ & $16,3 \%$ \\
\hline $\begin{array}{l}\text { Conectores concesivos: aun así, con } \\
\text { todo, de cualquier manera, etc. }\end{array}$ & $15,3 \%$ & $28,3 \%$ & $26,5 \%$ & $29,9 \%$ \\
\hline $\begin{array}{l}\text { Conectores reformuladores: dicho } \\
\text { de otro modo, mejor dicho, etc. }\end{array}$ & $17,4 \%$ & $29,7 \%$ & $25,1 \%$ & $27,8 \%$ \\
\hline $\begin{array}{l}\text { Conectores ejemplificativos: así, } \\
\text { por ejemplo, verbigracia, etc. }\end{array}$ & $26,5 \%$ & $32,1 \%$ & $24,1 \%$ & $17,3 \%$ \\
\hline $\begin{array}{l}\text { Conectores rectificativos: más bien, } \\
\text { mejor dicho, etc. }\end{array}$ & $20,3 \%$ & $33,2 \%$ & $27,4 \%$ & $19,1 \%$ \\
\hline $\begin{array}{l}\text { Conectores recapitulativos: al fin y } \\
\text { al cabo, en definitiva, en resumen, etc. }\end{array}$ & $20,6 \%$ & $26,4 \%$ & $33,3 \%$ & $19,7 \%$ \\
\hline $\begin{array}{l}\text { Conectores de ordenación: antes de } \\
\text { nada, en primer lugar, finalmente, etc. }\end{array}$ & $25,8 \%$ & $33,2 \%$ & $20,8 \%$ & $20,2 \%$ \\
\hline $\begin{array}{l}\text { Conectores de apoyo } \\
\text { argumentativo: pues bien, dicho esto, en } \\
\text { vista de aquello, etc. }\end{array}$ & $19,1 \%$ & $29,4 \%$ & $32,8 \%$ & $18,7 \%$ \\
\hline $\begin{array}{l}\text { Conectores de digresión: por cierto, } \\
\text { a propósito, dicho sea de paso, etc. }\end{array}$ & $22,1 \%$ & $34,1 \%$ & $25,9 \%$ & $17,9 \%$ \\
\hline TOTAL & 235,1 & 382,4 & 340,4 & 243 \\
\hline
\end{tabular}

Fuente: Elaboración propia

La comparación de los resultados de ambos cuadros parece sugerir que la metodología desarrollada por el modelo EPI es más eficiente para la asimilación y el uso de los conectores discursivos por parte de los aprendices de una L2. Estos resultados parecen mostrar que el hecho de que los propios alumnos puedan ir confirmando su propio progreso mediante la autoevaluación estimula el aprendizaje.

Ciertamente, hay que recalcar que las diferencias son bastante reducidas en porcentaje y que no parecen afectar del mismo modo a todas las tipologías. En este sentido, parece que lo más llamativo es el señalar que las ventajas del modelo EPI se muestran tanto en el nivel alto como en el nivel medio de dominio. Sin embargo, existen algunos tipos en los que parece que el modelo AICLE está al mismo nivel de resultados; incluso, en algún que otro tipo, llega superar a las evaluaciones realizadas en el modelo EPI. 
Parece evidente, por tanto, que los alumnos que han seguido la metodología EPI se han beneficiado de la práctica de la evaluación formativa, lo que supone que han asumido el hecho de que el aprendizaje de las lenguas ha de realizarse en pleno contacto de la L1 con el resto de las L2. Esto implica que este grupo ha asimilado la idea de que el saber competencial necesita del "saber ser", "saber estar" y "saber hacer" para que una competencia sea completa (Mallol y Alsina, 2017). Es decir, que estos alumnos «han percibido el TIL [EPI] como un enfoque que les conduce a saber más, pero también a saber utilizar y aplicar mejor lo aprendido, y a ser más competentes en el ámbito lingüístico» (Mallol y Alsina, 2017, p. 50).

Por el contrario, el resultado algo menos satisfactorio arrojado por el modelo AICLE puede deberse a que en dicho modelo no ha existido la interdependencia y transversalidad entre las áreas lingüísticas, no se ha practicado el translenguaging como estrategia para la corrección entre aprendizajes.

\section{CONCLUSIONES}

El propósito de este trabajo ha sido el comparar los resultados didácticos de dos grupos de aprendices de L2 siguiendo las dos metodologías didácticas más generalizadas hoy. No nos ha sorprendido el hecho de que, en el tema del dominio de los conectores discursivos en una lengua extranjera, el grupo que ha recibido una acción pedagógica globalizada por medio de la metodología EPI haya obtenido mejores resultados que el grupo que ha seguido la metodología AICLE. Hay que señalar que las diferencias en los resultados sugieren lo que estamos afirmando, pero que las diferencias son tan exiguas que sería conveniente realizar estudios con mayor profundidad para asegurar con rotundidad la superioridad de una metodología sobre la otra.

De los datos aportados tras las respectivas evaluaciones, tanto por medio de rúbricas como a través de otras herramientas, podemos deducir que los primeros cuentan con un mayor nivel de agentividad, entendiendo por esta la capacidad del aprendiz para tomar las riendas de su propio aprendizaje, fomentar su competencia lingüística e incrementar su capacidad de aprendizaje personal.

Parece relevante señalar el hecho de que los alumnos evaluados desde EPI perciben el aprendizaje como un reto significativo que los alienta a desarrollar habilidades, conocimientos y competencias, pues son agentes de su propio proceso de interiorización de los contenidos expuestos en el aula. 
En definitiva, los aprendices que han recibido la explicación de los conectores discursivos con la metodología EPI han tomado conciencia de la existencia de un marco común de estructuras y estrategias lingüísticas y han practicado el empoderamiento, entendido como «el proceso de concienciación que da cuenta al estudiante de sus capacidades y potencialidades y la relación de estas con el mundo que le rodea» (Torres de Márquez, 2009, p. 90).

\section{REFERENCIAS}

Andrade, Heidi. "Using Rubrics to promote thinking and learning in Education". Educational Leadership, 57.5 (2000): 13-18.

Berenguer Martín, Jorge. IX Liceum Ogólnokształcące, Polonia. "La Casa de Bernarda Alba. Unidad AICLE”. [Power Point Slider]. Asignatura de literatura española, sección bilingüe en español, en Polonia. 4 de diciembre de 2013 https://es.slideshare.net/jorgeberenguermartin/la-casade-bernarda-alba-unidad-aicle

Bludau, M. "Zur Stand des bilingualen Unterrichts in der Bundesrepublik Deutschland”. Neusprachliche Mitteilungen, 4 (1996): 208-215.

Council of Europe. Common European Framework of Reference for Languages: Learning Teaching, Assessment, Strasbourg: Language policy Unit, 2001.

Coyle, Do. "Supporting students in content and language integrated context: planning for effective classroom". En Masih, J. (Ed.). Learning through a foreign language: models, methods and outcomes. London: Center for Information on Language Teaching and Research (1999): 46-62.

Coyle, Do. "Content and Language Integrated Learning. Motivating Learners and Teachers". 2006. <http://blocs.xtec.cat/clilpractiques1/files/2008/11/sircoyle.pdf>

Cummins, Jim. "Rethinking monolingual instructional strategies in multilingual classrooms". Canadian Journal of Applied Linguistics, 10.2 (2007): 221-240.

Engeström, Yrjö "From Design Experiments to Formative Interventions". Theory and Psychology, 21.5 (2011): 3-24. https://doi.org/10.1177/0959354311419252 
Esteve, Olga. "La interacción en el aula desde el punto de vista de la construcción de conocimientos: un planteamiento didáctico”. En Salaberri, S. (Ed.). La lengua, vehiculo cultural multidisciplinar. Madrid: Ministerio de Educación, Cultura y Deporte, 2002.

Esteve, Olga, Ernesto Martín y Encarna Atienza. “The Integrated Plurilingual Approach: A didactic model providing guidance to Spanish schools for reconceptualizing the teaching of additional languages". Sociocultural Theory, 4.1 (2017): 1-24. https://doi.org/10.1558/1st.32868

Eurydice. Content and Language Integrated Learning (CLIL) at School in Europe. Brussels: European Unit, 2006.

Fuentes, Catalina. "La gramática discursiva; niveles, unidades y planos de análisis", Cuadernos AISPI, 2 (2013): 15-33.

Galperin, Piotr. "Stage by stage as a method of psychological investigation". Journal of Russian and East European Psychology, 30.4 (1992): 69-80. https://doi.org/10.2753/RPO1061-0405300460

Garrido, María del Camino. "Los conectores pragmáticos en la enseñanza de ELE: argumentación y relevancia". En Actas del X Congreso Internacional de ASELE. Cádiz: Servicio de Publicaciones de la Universidad de Cádiz, (2000): 323-330.

Gatica, Florina y Teresita del Niño Jesús Uribarren. “¿Cómo elaborar una rúbrica?”, Investigación en Educación Médica, 2.5 (2013): 61-65. http://dx.doi.org/10.1016/S2007-5057(13)72684-X

Graddol, David. (2006). English next. London: British Council, 2006.

Hablacultura. "Canciones para aprender español <20 de abril >". Hablacultura. Recursos para estudiantes y profesores de español. 20 de abril de 2018. https://hablacultura.com/20-de-abril/\#comments

Halliday, Michael Alexander. Language and Social Semiotic: the social interpretation of language and meaning. Baltimore: University Park Press, 1978.

Krashen, Stephen. Principles and practice in second language acquisition. Oxford: Pergamon Press, 1982.

Krashen, Stephen. The Input hypothesis: issues and implications, New York: Longman, 1985.

Longolia. "Los conectores discursivos". Longolia. Supérate con los idiomas. 2020.

https:/ / espanol.lingolia.com/es/vocabulario/conectores-discursivos

Llinares, Ana.; Tom Morton y Rachel Whittaker. The Role of Languages in CLIL. Cambridge:

Cambridge University Press, 2012. 
Mallol, Cristina y Miquel Alsina. "Enfoque plurilingüe integrado: una experiencia educativa interdisciplinar en el tratamiento integrado de las lenguas en la ESO”. Revista de Lingüística y Lenguas Aplicadas, 12 (2017): 45.53 http://dx.doi.org/10.4995/rlyla.2017.6547

Marsh, David. CLIL/EMILE, The European dimensions: actions, trends, and foresight potential. Brussel: European Commision, 2002.

Martí, Manuel y Sara Fernández. Los marcadores discursivos. Edinumen: Madrid, 2013.

Martín Ernesto. "Un modelo de enfoque plurilingüe en la enseñanza de la lengua en la escuela", Minguaran Arena, 5 (2014): 47-66.

Mehisto, Peeter; David Marsh y María Jesús Frigols. Uncovering CLIL, Content and Language Integrated learning in Bilingual and Multilingual Education. Oxford: Macmillan, 2008.

Meléndez, Carlos. (2007). "Esp. afortunadamente, desgraciadamente / fr. heureusement, malheureusement: esbozo contrastivo de dos pares de partículas modales”, en Interlingüistica, 17 (2007): 737-747.

Moate, Josephine. "Reconceptualising the role of talk in CLIL", Journal of Applied LanguageStudies, 5.2 (2011): 17-35.

Monereo, Carles. "La evaluación del conocimiento estratégico a través de tareas auténticas", Revista de Pensamiento Educativo, 32 (2003): 71-89.

Negueruela, Eduardo. "Beliefs as conceptualizing activity: A dialectical approach for the second $\begin{array}{llll}\text { language } & \text { classroom”. } & \text { System, } & 39\end{array}$ http://dx.doi.org/10.1016/j.system.2011.07.008

Piccardo, Enrica. From communicative to Action-Oriented: a Research Pathway. Ontario: Ministry of Education, 2014

Torres, Analí. (2009). "La educación para el empoderamiento y sus desafíos". Sapiens. Revista Universitaria de Investigación, 10.1 (2009): 89-108.

Van Lier, Leo. "Action-based teaching, autonomy and identity". Innovation in Language Learning and Teaching, 1.1 (2007): 46-65.

Vigotsky, Lev. Mind and Society: The Development of Higher Psychological Process. Cambridge: Harvard University Press, 1978. 
Wolff, Dieter y David Marsh. Bridging the gap between school and working life. Frankfurt: Peter Lang, 2007.

Wylie, Caroline y Christine Lyon, C. Using the Formative Assessment Rubrics, Reflection and Observation Tools to Support Professional Reflection on Practice, Educational Testing Service Commissioned by Formative Assessment for Teachers and Students (FAST) State Collaborative on Assessment and Student Standards (SCASS) of the Council of Chief State School Officers (CCSSO), 2016. 